\title{
Mock-Chebyshev Noktalarında Vandermonde Matrisinin Bir Uygulaması: Kovid-19 Vaka Tahmini
}

\author{
B. Ali İbrahimoğlu ${ }^{1 *}$

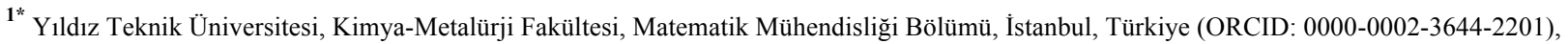 \\ bibrahim@yildiz.edu.tr
}

(İlk Geliş Tarihi 2 Aralık 2020 ve Kabul Tarihi 14 Ocak 2021)

(DOI: $10.31590 /$ ejosat.834950)

\begin{abstract}
ATIF/REFERENCE: İbrahimoğlu, B. A. (2021). Mock-Chebyshev Noktalarında Vandermonde Matrisinin Bir Uygulamas1: Kovid-19 Vaka Tahmini. Avrupa Bilim ve Teknoloji Dergisi, (21), 172-180.
\end{abstract}

Öz

Birbirinden farklı interpolasyon noktalarında (veya nodlarında) verilen datalar kullanılarak, polinom interpolasyon problemi monomial bazlar ile ifade edilebilir. Bu ifade, Vandermonde matrisi ile beraber bir lineer denklem sistemi oluşturur. Reel sayılarda verilen interpolasyon noktaları için bu noktaların sayısı çok küçük değilse; Vandermonde matrisi genel olarak kötü durumludur ve bu kötü durumluluk bu matris ile çalışmanın zorluklarından biridir. Bu kötü durumluluğun derecesi, interpolasyon noktalarının dağılımına bağlı olarak epeyce farklılık gösterebilir. Bu bağlamda, noktalarının dağılımı eşit aralıklı olmaktan epey uzak olan Chebyshev nodlarının kullanılması genelde tavsiye edilmektedir. Ancak, bu takdirde, deneysel verilerin sadece eşit aralıklı noktalarda mevcut olması durumunda ne yapılacağı problemi ortaya çıkar. Bu durumda, polinom interpolasyonunda iyi bilinen şu sorun oluşur: ele alınan fonksiyon interpolasyon aralığının her yerinde analitik olsa bile, eşit aralıklı noktalardaki interpolasyon polinomları yakınsamayabilir. Bu sorunun sebebi, Runge olgusu olarak bilinir. İnterpolasyon işlemlerinin Chebyshev nodlarında en iyi sonucu verme avantajından faydalanarak, Runge olgusunun üstesinden gelmenin en iyi yollarından biri, polinom interpolasyonunda mockChebyshev noktalarını kullanmaktır. Eşit aralıklı noktaların genişçe bir kümesinden seçilen bu noktalar asimptotik olarak Chebyshev noktalarının dağılımını takip ederler. Bu noktaların hesaplanması konusunda, literatürde çok az sayıda çalışma bulunmaktadır. Bu çalışmada, mock-Chebyshev noktalarını hesaplayan hızlı bir algoritma tanıtılmaktadır. Bu algoritma ile elde edilen noktalar kullanılarak, Kovid-19 vakalarını tahmin etmek için Vandermonde matrisinin bu noktalardaki bir uygulaması verilmektedir. İnterpolasyon noktalarının dağılımının Vandermonde matrisinin durumu açısından rolü ayrıca değerlendirilmiştir.

Anahtar Kelimeler: Vandermonde matrisi, Mock-Chebyshev interpolasyon, Nod dağılımları.

\section{An Application of the Vandermonde matrix with the mock- Chebyshev nodes: Estimating Covid-19 Cases}

\begin{abstract}
The polynomial interpolation problem through data given at a set of distinct interpolation points (or nodes) can be expressed in the monomial basis. This gives rise to a linear system of equations with a Vandermonde matrix. A difficulty with a Vandermonde matrix is that this matrix is generally quite ill-conditioned when the nodes are real and the number of points is not very small. This illconditioning may vary reasonably with the distribution of the points. The general recommendation is to use the highly non-uniform Chebyshev nodes, but the problem remains what to do if experimental data is available only at equally spaced points. In this case, polynomial interpolation has the well-known drawback that polynomial interpolants in equally spaced points might not converge, even if the considered function is analytic anywhere on the interval. The reason of this fact is termed the Runge phenomenon. By taking advantage of the optimality of the interpolation processes on Chebyshev nodes, one of the best strategies to defeat the Runge phenomenon is to use the mock-Chebyshev points for polynomial interpolation, which asymptotically follow the Chebyshev
\end{abstract}

*Sorumlu Yazar: bibrahim@yildiz.edu.tr 
distribution and they are selected from a large set of equispaced nodes. Yet, little literature exists on the computation of these points. A fast algorithm for computing the mock-Chebyshev nodes is introduced. Using the points obtained by the algorithm, an application of the Vandermonde matrix with these points is provided in order to estimate Covid-19 cases. We also consider the role of distribution of the interpolation points in terms of the conditioning of the Vandermonde matrix.

Keywords: Vandermonde matrix, Mock-Chebyshev interpolation, Node distributions.

\section{Giriş}

Nümerik analizde önemli bir yeri olan yaklaşım teorisi, Uygulamalı Matematik ve Hesaplamalı Bilimlerde yaygın bir şekilde kullanılmaktadır. Bazı fiziksel davranışları tanımlayan fonksiyon ve büyüklüklerin yaklaşımlarının bulunması bilimsel hesaplamanın bir parçasıdır. Görüntü işleme, materyal tasarımı, konuşma analizi, video sinyal filtreleme, kuyruk problem, nanoteknoloji, robotik, matematiksel finans, sinir ağları ve ăg trafiği bunlardan bazılarıdır. Bazı davranışları yaklaşık olarak temsil eden basit bir matematik fonksiyonunu bulmanın iki zorluğu vardır. Bunlardan biri, çalışılan davranışın kapalı bir matematik formülü ile ifade edilememesi; diğeri ise, elde edilen formülün herhangi bir uygulamada pratik olarak kullanılamayacak kadar karmaşık olmasıdır. Her iki durum için bu temsil fonksiyonu basit bir yapıda olması gerekmektedir. $\mathrm{Bu}$ durumda, hangi matematik fonksiyonlarının basit yapıda olduğu sorusu ortaya çıkar. Uygulamada en hızlı ve en basit fonksiyonlar polinomlardır. Çünkü, polinomlar sadece hızlıca yapılabilen temel işlemler olan toplama ve çarpma işlemlerini kullanırlar. $\mathrm{Bu}$ yüzden, basit ve kullanışlı bir yaklaşım yöntemi olan polinom interpolasyon konusunun geçmişten günümüze yoğun bir şekilde çalışıldığ ve bu yöntemin matematiksel modellemede sıklıkla kullanıldığı görülmektedir.

Birbirinden farklı interpolasyon noktaları ve bu noktalarda verilen datalar kullanılarak, polinom interpolasyon problemi çeşitli polinom bazlarıyla ifade edilebilir. Bunların her biri interpolasyon şartlarını sağlayan çeşitli avantaj veya dejavantajlara sahip farklı lineer sistemler üretirler. Burada, standart tek terimli monomial bazları, bölünmüş farkları kullanan Newton bazlarını, Lagrange bazlarını veya herhangi bir ortogonal polinomunun bazlarının seçilmesini örnek olarak verebiliriz. Kullanılan interpolasyon noktaları aynı kalmak şartıyla bir ve yalnız bir polinom elde edilebildiğinden, farklı bazların kullanılması aynı polinomun farklı formlarda üretilmesi sonucunu verir. Bununla beraber, baz seçimi matematiksel açıdan bir fark oluşturmasa da interpolasyon polinomunu sayısal olarak hesaplarken problemin durumunu önemli ölçüde etkiler.

Birbirinden farklı $x_{0}, \ldots, x_{n}$ interpolasyon noktalarında (veya nodlarında) verilen $f_{0}, \ldots, f_{n}$ dataları kullanılarak, interpolasyon polinomu monomial bazlarda $1, x, \ldots, x^{n}$ fonksiyonlarının bir lineer kombinasyonu şeklinde ifade edilebilir. Bu durumda Vandermonde matrisi olarak adlandırılan katsayllar matrisi ile beraber bir lineer denklem sistemi elde edilir. İnterpolasyon noktaları birbirinden farklı olduğu sürece elde edilen lineer sistemdeki Vandermonde matrisinin determinantı sıfırdan faklı olur ve böylece $f_{0}, \ldots, f_{n}$ datalarının herhangi bir seçimi için lineer sistemi her zaman tek bir çözüme sahip olur. Ancak, interpolasyon noktaları reel say1 ve bu noktaların sayısı çok küçük olmadığında, Vandermonde matrisi genelde kötü durumludur ve böylece oluşan lineer denklem sisteminin çözümünde hatalı sonuç elde edilmesi muhtemeldir.
İnterpolasyon polinomunun hesaplanması için, problemin ifadesinin iyi durumda olması nümerik açıdan arzu edilen bir durumdur. $\mathrm{Bu}$ bağlamda, interpolasyon noktalarının dağılımı hem elde edilecek yaklaşımın kalitesi hem de bu yaklaşımın nümerik olarak hesaplanmasında çok önemli bir role sahiptir [7]. Bunu göstermek için, interpolasyonda eşit aralıklı noktaları ve noktalarının dağılımı eşit aralıklı olmaktan epey uzak olan Chebyshev noktalarını ele almak yeterlidir. Eşit aralıklı noktalar kullanıldığında, sürekli fonksiyonların çoğu için interpolasyon polinomu düzgün yakınsamaz. Buna standart örnek olarak, $[-1,1] \quad$ aralığında $f(x)=\left(25 x^{2}+1\right)^{-1} \quad$ Runge fonksiyonunun polinom interpolasyonu verilmektedir. $\mathrm{Bu}$ fonksiyon için interpolasyon polinomu eşit aralıklı noktalar kullanılarak oluşturuluyorsa, interpolasyon aralığının sınırlarına doğru gidildikçe çok büyük salınımlar görülecek ve böylece interpolasyon polinomu Runge fonksiyonuna yakınsamayacaktır [10]. $\mathrm{Bu}$ durum Runge olgusu olarak adlandirılır. Burada sorunun kaynağı; Runge fonksiyonunun her reel $x$ değişkeni için analitik olması sebebiyle sorunsuz görünmesine rağmen, kompleks düzlemin sanal ekseninde ( $x= \pm \pi i / 5$ 'te) bir tekil komşuluğa sahip olmasıdır. Eşit aralıklı noktalarda interpolasyon için bir başka sorun problemin kötü durumlu olmasıdır. Örneğin; $[-1,1]$ aralığında eşit aralıklı noktalar kullanılarak, $f(x)=e^{x}$ gibi bir tekil komşuluğu bulunmayan ve aralığın her yerinde analitik olan fonksiyon için oluşturulan interpolasyon polinomu, bu fonksiyonuna teorikte yakınsaması gerekirken pratikte bilgisayarda nümerik olarak interpolasyon polinomu hesaplanırken oluşacak yuvarlama hataları problemin eşit aralıklı noktalarda kötü durumlu olması sebebiyle $n \rightarrow \infty$ iken yakınsak sonuç elde edilmesine engel olacaktır.

Buna karşın, interpolasyon aralığının sınırlarına doğru gittikçe, önemli ölçüde fazla interpolasyon noktasına sahip olan Chebyshev noktalarında durum tamamen farklıdır. Örneğin, Runge fonksiyonu için Chebyshev noktalarında interpolasyon polinomu geometrik olarak yakınsar ve problem iyi durumludur. Dahası tüm analitik fonksiyonlar için bu durum geçerlidir. İnterpolasyon polinomunun eşit aralıklı noktalardaki davranışları ile Chebyshev noktalarındaki davranışları kıyaslandığında çok farklı sonuçlar vermesinin temel sebebi noktaların dağılımıdır. Dolayısıyla iyi sonuç elde etmek için izlenecek yol, polinom interpolasyonunda asimtotik olarak Chebyshev noktalarının dağılımını takip eden noktalar kümesinin kullanılmasıdır [11].

Ancak pek çok bilimsel araştırmada veriler genelde eşit aralıklı noktalarda elde edilir. Örneğin bir deney veya gözlemde ölçümler genellikle eşit aralıklı olacak şekilde yapılır. Bu eşit aralıklı verileri kullanarak basit ve kullanışlı olan polinom interpolasyonu ile bir yaklaşım elde edilmek istendiğinde; hem Runge olgusundan kaçınmak hem de Chebyshev noktalarının kullanılmasına benzer şekilde iyi bir sonuç elde etmek için, takip edilebilecek en iyi stratejilerden biri son zamanlarda geliştirilen mock-Chebyshev noktalarını kullanmaktır [1, 2]. Eşit aralıklı noktaların genişçe bir kümesinden seçilerek elde edilen bu noktalar asimptotik olarak Chebyshev noktalarının dağılımını 
takip ederler. Ancak bu noktaların hesaplanması için gereken işlem yükü fazladır ve bu konuda literatürde yeteri kadar çalışma yoktur. Fakat $[-1,1]$ aralığında eşit aralıklı noktaların sayısının serbestçe seçilebildiği durumda, mock-Chebysev noktalarını hesaplayan hızlı bir algoritma yakın zamanda yayınlanan [8] makalesinde verilmiştir. Bu sonuçtan hareketle, çalışma aralığını reel eksende $[a, b]$ aralığına genişleten bir algoritma bu çalışmada önerilmiştir. Bu algoritma ile elde edilen noktaların performansını test etmek için, bu noktalar polinom interpolasyonunda kullanılmıştır. Bunun için Kovid-19 vakalarını ara günlerde tahmin eden interpolasyon polinomu Vandermonde matrisi kullanılarak elde edilmiştir. Elde edilen sonuçlar, eşit aralıklı noktalar kullanılması durumunda elde edilen sonuçlar ile karşılaştırılmıştır.

\section{Polinom İnterpolasyonu}

\subsection{Vandermonde Sistemi}

Birbirinden farklı $x_{0}, \ldots, x_{n} \in[a, b] \subset \mathbb{R}$ noktaları (veya nodları) ve bu noktalardaki $f_{0}, \ldots, f_{n}$ verileri (veya fonksiyon değerleri) kullanılarak,

$$
p_{n}(x)=\sum_{i=0}^{n} a_{i} x^{i}
$$

olacak şekilde

$$
p_{n}\left(x_{j}\right)=f_{j}, \quad j=0, \ldots, n
$$

interpolasyon şartlarını sağlayan, derecesi en fazla $n$ olan tek bir interpolasyon polinomu vardir. $\mathrm{Bu} \quad p(x)$ polinomunun $a_{0}, \ldots, a_{n}$ katsayıları

$$
\left[\begin{array}{ccccc}
1 & x_{0} & x_{0}^{2} & \cdots & x_{0}^{n} \\
1 & x_{1} & x_{1}^{2} & \cdots & x_{1}^{n} \\
1 & x_{2} & x_{2}^{2} & \cdots & x_{2}^{n} \\
\vdots & \vdots & \vdots & \ddots & \vdots \\
1 & x_{n} & x_{n}^{2} & \cdots & x_{n}^{n}
\end{array}\right]\left[\begin{array}{c}
a_{0} \\
a_{1} \\
a_{2} \\
\vdots \\
a_{n}
\end{array}\right]=\left[\begin{array}{c}
f_{0} \\
f_{1} \\
f_{2} \\
\vdots \\
f_{n}
\end{array}\right]
$$

şeklinde Vandermonde sistemi olarak adlandırılan ve $V \mathbf{a}=\mathbf{f}$ şeklinde gösterilecek $n+1$ boyutlu bir lineer denklem sisteminin çözümü ile hesaplanabilir. Burada oluşan $(n+1) \times(n+1)$ boyutundaki kare matris katsayılar matrisidir. Bu katsayılar matrisine Vandermonde matrisi denir ve aşağıdaki şekilde gösterilir:

$$
V=V_{n}\left(x_{0}, \ldots, x_{n}\right)=\left[\begin{array}{ccccc}
1 & x_{0} & x_{0}^{2} & \cdots & x_{0}^{n} \\
1 & x_{1} & x_{1}^{2} & \cdots & x_{1}^{n} \\
1 & x_{2} & x_{2}^{2} & \cdots & x_{2}^{n} \\
\vdots & \vdots & \vdots & \ddots & \vdots \\
1 & x_{n} & x_{n}^{2} & \cdots & x_{n}^{n}
\end{array}\right] .
$$

Burada oluşan $V \mathbf{a}=\mathbf{f}$ lineer denklem sistemi ancak ve ancak $\operatorname{det} V \neq 0$ ise tek bir çözüme sahiptir. Birbirinden farklı $x_{0}, x_{1}, \ldots, x_{n}$ sayıları için Vandermonde matrisinin determinant

$$
\begin{aligned}
\operatorname{det} V_{n}\left(x_{0}, \ldots, x_{n}\right) & =\operatorname{det}\left[\begin{array}{ccccc}
1 & x_{0} & x_{0}^{2} & \cdots & x_{0}^{n} \\
1 & x_{1} & x_{1}^{2} & \cdots & x_{1}^{n} \\
1 & x_{2} & x_{2}^{2} & \cdots & x_{2}^{n} \\
\vdots & \vdots & \vdots & \ddots & \vdots \\
1 & x_{n} & x_{n}^{2} & \cdots & x_{n}^{n}
\end{array}\right] \\
& =\prod_{0 \leq j \leq n}\left(x_{i}-x_{j}\right)
\end{aligned}
$$

eşitliğini sağlar. Burada, $x_{0}, x_{1}, \ldots, x_{n}$ sayıları birbirinden farklı olduğu sürece $\operatorname{det} V \neq 0$ olduğu aşikardır. Böylece, $f_{0}, \ldots, f_{n}$ datalarının herhangi bir seçimi için $V \mathbf{a}=\mathbf{f}$ lineer denklem sisteminin her zaman tek bir çözümü vardır.

\subsection{Polinom İnterpolasyonunda Hatanın Sınırları}

Birbirinden farklı $x_{0}, x_{1}, \ldots, x_{n} \in[a, b]$ noktalarında bir $f(x)$ fonksiyonunu interpole eden ve derecesi en fazla $n$ olan interpolasyon polinomu $p_{n}(x)$ olsun. $\mathrm{Bu}$ durumda, $f(x) \in C^{(n+1)}([a, b])$ ise, oluşan hata

$$
f(x)-p_{n}(x)=\frac{f^{(n+1)}\left(\xi_{x}\right)}{(n+1) !} \prod_{j=0}^{n}\left(x-x_{j}\right)
$$

şeklinde ifade edilir. Burada, $\xi_{x} \in\left(x_{0}, x_{n}\right)$ bir sayı, $f^{(n+1)}\left(\xi_{x}\right)$ ise $f(x)$ fonksiyonunun $(n+1)$-inci mertebeden türevinin bu sayıdaki değeridir. Fakat, genelde $\xi_{x}$ sayısı tam olarak bilinmez ve bu durumda hata hakkında bir tahmin yapılmasını sağlayan aşağıdaki hata formülü önemlidir [3]:

$$
\begin{aligned}
\max _{x \in[a, b]}\left|f(x)-p_{n}(x)\right| \\
\leq \max _{x \in[a, b]}\left(\frac{\left|f^{(n+1)}(x)\right|}{(n+1) !}\right) \max _{x \in[a, b]} \prod_{j=0}^{n}\left|x-x_{j}\right|
\end{aligned}
$$


Burada, $f(x)$ fonksiyonu problem tarafindan sabitlenmiş olduğundan, hatanın üst sınırı üzerinde meydan gelebilecek herhangi bir etki; Denklem (6)'daki Cauchy hata çarpanı olarak adlandirılan $Q(x)=\prod_{j=0}^{n}\left(x-x_{j}\right)$ ifadesinden dolayı, sadece $x_{j}$ noktalarının seçimine bağlı olacaktır. İnterpolasyon noktaları $(n+1)$-inci dereceden Chebyshev polinomunun kökleri olacak şekilde seçilirse, $\max _{x \in[-1,-1]} \prod_{j=0}^{n}\left|x-x_{j}\right|$ ifadesi minimum olur ve bu değer $\frac{1}{2^{n}}$ 'dir [3]. Bu noktalara Chebyshev noktaları denir ve

$$
x_{j}=\cos \left(\frac{2 j+1}{2 n+2} \pi\right), j=0, \cdots, n
$$

şeklinde tanımlanırlar. Cauchy hata çarpanının mutlak değerinin maksimum değerini $\frac{1}{2^{n-1}}$ ile minimuma yakın yapan bir başka noktalar kümesi ise, Chebyshev polinomunun ektremumları olarak adlandırılan ve tanım aralığının uç noktaları olan \pm 1 sayılarını içermeleriyle Chebyshev noktalarından ayrışan Chebyshev-Lobatto noktalarıdır ve bu noktalar aşağıdaki şekilde tanımlanır:

$$
x_{j}=\cos \left(\frac{j}{n} \pi\right), j=0, \cdots, n .
$$

Eğer noktalar eşit aralıklı olarak,

$$
x_{j}=-1+\frac{2 j}{n}, j=0, \cdots, n
$$

şeklinde seçilirse, Chebyshev-Lobatto noktalarının aksine, $\max _{x \in[-1,1]}|Q(x)|$ ifadesi $\frac{(2 n) !}{2^{n} n^{n+1} n !}$ gibi büyük değerli alt sınırı ile, tahmin edilebileceği gibi, kötü sonuç verecektir. Bu duruma standart örnek olarak, $[-1,1]$ aralığında eşit aralıklı noktalarda Runge fonksiyonu $f(x)=\frac{1}{1+(5 x)^{2}} \quad$ 'nin polinom interpolasyonu verilir.

\subsection{Vandermonde Sisteminin Nümerik Durumu}

Uygulamalı matematik ve nümerik analizde pek çok problem, bir lineer denklem sistemine indirgenerek çözülebilir. Gerek verilerin elde edilmesi esnasında yapılan ölçme hataları gerekse çözüm esnasında yapılan yuvarlama hataları sebebiyle, problemin modellenmesi sonucu oluşan lineer denklem sisteminin çözümünde hatalı sonuç elde edilmesi muhtemeldir. Polinom interpolasyonunda, bu hataların $V \mathbf{a}=\mathbf{f}$ denklem sisteminin çözümü üzerindeki etkisi ||.|| uygun bir matris normu olmak üzere,

$$
\kappa(V)=\|V\|\left\|V^{-1}\right\|
$$

durum sayısı ile aşağıdaki gibi tahmin edilebilir [9]:

1) $V \mathbf{a}=\mathbf{f}$ lineer denklem sisteminin sağ taraf değerlerinde (f vektöründe) küçük hatalar sonucu oluşan yeni sistem $V \tilde{\mathbf{a}}=\tilde{\mathbf{f}}$ olsun. Bu durumda,

$$
\frac{\|\mathbf{a}-\tilde{\mathbf{a}}\|}{\|\mathbf{a}\|} \leq \kappa(V) \frac{\|\mathbf{f}-\tilde{\mathbf{f}}\|}{\|\mathbf{f}\|}
$$

eşitsizliği elde edilir. Bu da, katsayılar vektörü a 'daki bağıl hatanın, $f_{0}, f_{1}, \ldots, f_{n}$ verilerinin oluşturduğu $\mathbf{f}$ vektöründeki bağıl hatanın $\kappa(V)$ katından daha fazla olamayacağını gösterir. Ayrıca, eğer $\kappa(V)$ durum sayısı küçük ise, $\mathbf{f}$ vektöründe yapılacak küçük değişikliklerin a vektöründe de küçük değişiklere yol açacağını ifade eder.

2) $V \mathbf{a}=\mathbf{f}$ lineer denklem sisteminin katsayılar matrisinde $(V$ de) küçük hatalar sonucu oluşan yeni sistem $\tilde{V} \tilde{\mathbf{a}}=\mathbf{f}$ olsun. $\mathrm{Bu}$ durumda,

$$
\frac{\|\mathbf{a}-\tilde{\mathbf{a}}\|}{\|\mathbf{a}\|} \leq \kappa(V) \frac{\|V-\tilde{V}\|}{\|V\|}
$$

eşitsizliği elde edilir. $\mathrm{Bu}$ da, katsayılar matrisi $V$ deki bağıl hatanın $\kappa(V)$ katının, katsayılar vektörü a 'daki bağıl hata için bir üst sınır olduğunu gösterir.

Kullanılan normdan bağımsız olarak durum sayısı minimum 1 'dir. Bu özellikteki matrise mükemmel durumlu matris denir. 
Eğer, durum sayısı $\kappa(V)$ büyük ise (veya hızlı bir şekilde büyüyorsa), $\quad V \mathbf{a}=\mathbf{f}$ lineer denklem sisteminde katsayılar matrisi $V$ 'de veya denklemin sağ tarafı olan f'de yapılan küçük değişiklikler, sonuç matrisi olan a 'da çok büyük değişikliğe sebep olur. $\mathrm{Bu}$ durumda, matris kötü durumlu olarak adlandirılır. Bunun tersi olarak, $V$ 'nin durum sayıs1 $\kappa(V)$ küçük ise (veya yavaş bir şekilde büyüyorsa), bu takdirde matris iyi durumlu olarak adlandırılır.

$A=\left[a_{i j}\right]_{n \times n}$ matrisi için burada kullanılan değişik matris normları aşağıdaki gibi tanımlanır:

(I) $\|A\|_{1}=\max _{1 \leq j \leq n} \sum_{i=1}^{n}\left|a_{i j}\right|, A$ matrisinin sütun normudur ve her bir sütunun elemanlarının mutlak değerlerinin ayrı ayrı toplamlarının maksimum değerine eşittir.

(II) $\|A\|_{\infty}=\max _{1 \leq i \leq n} \sum_{j=1}^{n}\left|a_{i j}\right|, A$ matrisinin satır normudur ve her bir satırın elemanlarının mutlak değerlerinin ayrı ayrı toplamlarının maksimum değerine eşittir.

(III) $\|A\|_{F}=\left(\sum_{i=1}^{n} \sum_{j=1}^{n}\left|a_{i j}\right|^{2}\right)^{1 / 2}, A$ matrisinin Frobenius normudur ve matrisin tüm elemanlarının mutlak değerlerinin kareleri toplamının kareköküne eşittir.

(IV) $\|A\|_{2}=\sqrt{\rho_{\max }\left(A^{T} A\right)}, A$ matrisinin spektral normudur ve $A^{T} A$ matrisinin en büyük öz değerinin kareköküne eşittir.

\subsubsection{Vandermonde Matrisinin Durum Sayıs}

Vandermonde matrisinin, $x_{0}, x_{1}, \ldots, x_{n}$ noktalarında ve $p$ normuna göre ( $p=1,2, \infty$, Frobenius ) durum sayısını,

$$
\begin{aligned}
\kappa_{p}\left(V_{n}\right) & :=\kappa_{p}\left(V_{n} ; x_{0}, \ldots, x_{n}\right) \\
& =\left\|V_{n}\left(x_{0}, \ldots, x_{n}\right)\right\|\left\|_{p}\right\| V_{n}^{-1}\left(x_{0}, \ldots, x_{n}\right) \|_{p}
\end{aligned}
$$

şeklinde gösterelim. Durum sayısı $\kappa_{p}\left(V_{n}\right)$ 'nin $x_{j}$ noktalarına ve kullanılan norma bağlı olduğu açıktır. Ancak, matris normları arasındaki bağıntılardan, $\kappa_{\alpha}(\cdot)$ ve $\kappa_{\beta}(\cdot)$ iki farklı matris normundaki durum sayılarını göstermek üzere, $c_{1}$ ve $c_{2}$ sabitleri kullanılarak

$$
c_{1} \kappa_{\alpha}(A) \leq \kappa_{\beta}(A) \leq c_{2} \kappa_{\alpha}(A), \quad A \in \mathbb{R}^{n \times n}
$$

eşitsizliği yazılabilir. Örneğin, $\kappa_{1}(\cdot)$ ve $\kappa_{\infty}(\cdot)$ için

$$
\frac{1}{n^{2}} \kappa_{1}(A) \leq \kappa_{\infty}(A) \leq n^{2} \kappa_{1}(A)
$$

eşitsizliği sağlanır. Böylece, $A$ matrisi, herhangi bir matris normunda kötü durumlu ise yukarıdaki $c_{1}$ ve $c_{2}$ sabitleri ölçüsünde diğer bir matris normunda da kötü durumlu olur.

Vandermonde matrisinin durum sayıs1, $x_{0}, x_{1}, \ldots, x_{n}$ reel sayıları için $n$ ye bağlı olarak üstel olarak artar ve bu üstel büyüme kötü durumlu bir matris olduğunu gösterir. Bunun en büyük sebebi, Vandermonde matrisini oluşturan monomial bazlardır. Çünkü yüksek dereceden $n$-inci monomialler birbirlerinden daha az ayırt edilebilir durumdadır. $\mathrm{Bu}$ ise, Vandermonde matrisinin sütunlarını neredeyse lineer bağımlı hale getirir. Gautschi [4] tarafından $[-1,1]$ aralığındaki reel sayılar için Vandermonde matrisinin sütun normundaki durum sayısının üst sınırının matrisin tersi bulunmadan

$$
\kappa_{1}\left(V_{n}\right) \leq \lim _{0 \leq i \leq n} \sum_{j=0}^{n} x_{j}^{i} \lim _{0 \leq i \leq n} \sum_{j=0, j \neq i}^{n} \frac{1+\left|x_{j}\right|}{\left|x_{i}-x_{j}\right|}
$$

şeklinde hesaplanabileceği gösterilmiştir. Dahası, eğer aralık $[-1,0]$ veya $[0,1]$ şeklinde ise (16) eşitsizliğinin eşitliğe dönüşeceği ve böylece durum sayısının doğrudan hesaplanabileceğini göstermiştir. Bunlara ilaveten, değişik nokta kümeleri için sütun normundaki durum sayısının büyüme formülleri Gautschi tarafından elde edilmiştir. Bunlardan bazıları aşağıdaki gibidir [5]:

$[-1,1]$ reel Chebyshev noktaları için,

$$
\kappa_{1}\left(V_{n}\right) \sim \frac{3^{\frac{3}{4}}}{4}(1+\sqrt{2})^{n+1}, n \rightarrow \infty
$$

ve $[-1,1]$ reel aralığındaki eşit aralıklı noktalar için,

$$
\kappa_{1}\left(V_{n}\right) \sim \frac{\sqrt{2}}{\pi}\left(e^{\frac{\pi}{4}} \sqrt{2}\right)^{n}, n \rightarrow \infty
$$

Ayrica, polinom interpolasyon probleminin ortonormal polinom bazlariyla ifade edilmesi durumumda elde edilecek Vandermonde benzeri matrisin durum sayısının çok azalacağı ve hatta spektral normda mükemmel durumlu bir matris olacağ 1 ilk kez Gautschi [6] tarafindan gösterilmiştir. $\mathrm{Bu}$ mükemmel 
durumluluk, Vandermonde matrisi için reel eksende değil, kompleks düzlemde noktaların birim çember üzerinde eşit aralıklı olacak şekilde seçilmesiyle gerçekleşir.

interpolasyonu verilir.

\subsection{Mock-Chebyshev Noktaları}

Polinom interpolasyonunda noktalarının dağılımı (noktaların geometrik yeri) çok önemlidir. Bazı nokta kümeleri ile optimale (en iyiye) yakın yaklaşımlar elde edilirken, bazılarıyla çok kötü (1raksak) sonuçlar elde edilebilir. Bu bağlamda, interpolasyon aralığının sınırlarına doğru gidildikçe önemli ölçüde fazla noktanın mevcut olduğu ve $[-1,1]$ aralığı için bu noktaların dağılımının $\frac{n}{\pi \sqrt{1-x^{2}}}$ yoğunluk fonksiyonuna sahip olduğu Chebyshev noktalarının interpolasyon noktaları olarak kullanılması tavsiye edilen bir durumdur. Gerek hata formülündeki Cauchy çarpanı gerekse durum sayısı $\kappa_{1}\left(V_{n}\right)$ 'nin Chebyshev noktalarındaki değerleri ile eșit aralıklı noktalardaki değerleri kıyaslandığında, interpolasyon polinomunda Chebyshev noktalarının kullanılmasının çok daha iyi sonuç vereceği açıktır. Dahası, polinom interpolasyonunda Chebyshev noktaları optimale yakın sonuç verirken, eşit aralıklı noktalar ise Runge olgusundan dolayı analitik fonksiyonlar için dahi rraksak sonuç verebilmektedir. Fakat bir gözlem veya ölçüm sırasında veriler genelde eşit aralıklı olacak şekilde elde edilir. $\mathrm{Bu}$ durumda, eşit aralıklı nokta dağılımının kötü sonuçlarından kaçınmak ve polinom interpolasyonunun hatasını azaltmak için son yıllarda geliştirilen mock-Chebyshev noktaları olarak adlandırılan iyi bir alternatif yöntem vardır. Bu yöntem, polinom interpolasyonunda kullanılacak verilerin eşit aralıklı genişçe bir kümesinden Chebyshev-Lobatto noktalarının dağılımı dikkate alınarak bir alt küme oluşturulmasına dayanır. Fakat bu lokal optimizasyon gerektirdiğinden, $O\left(n^{3}\right)$ mertebeden işlem yükü ile maliyetli bir yöntemdir. Ancak bu işlem yükünü $O(n)$ mertebesine indiren aşağıdaki algoritma, mock-Chebyshev noktalarının elde edilmesinde iyi bir alternatif sunmaktadır.

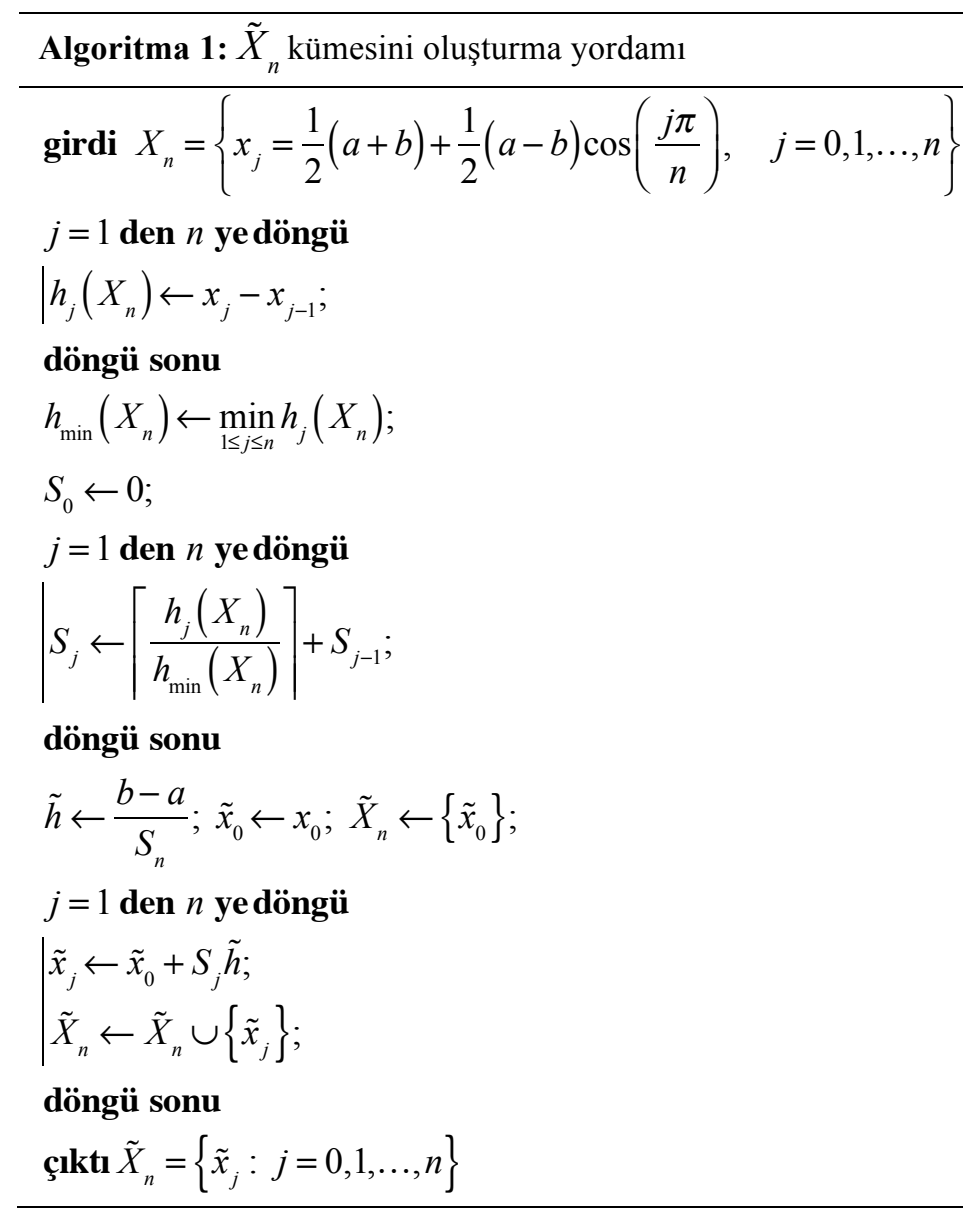

\section{Sayısal Sonuçlar ve Tartışma}

Bu bölümde, Algoritma 1 ile elde ettiğimiz noktaların performansını ölçmek için 2020 yılında meydana gelen koronovirüs pandemisinden en çok etkilenen ülkelerden biri olan İtalya'nın 18 Mart 2020 ile 23 Nisan 2020 arası Worldometers'in [12] verilerine göre, doğrulanmış günlük koronavirüs vaka sayılarını kullanarak polinom interpolasyonları elde edelim. Bunun için, öncelikle, bu tarihler arasındaki 37 günlük verilerden 10 tanesini seçerek interpolasyon polinomu ile bir yaklaşım elde edip sonra elde ettiğimiz polinom ile kullanmadı̆̆ımız günlerin verilerini tahmin ederek yapılan hatayı hesaplayalım.

Öncelikle, bu 10 günlük veriyi 37 günlük veri içerisinden 18 Mart 2020 tarihini ilk gün, 23 Nisan 2020 tarihini ise 37-inci gün olarak kabul edip ilk günden başlamak üzere 4'er gün arayla eşit aralıklı olacak şekilde Tablo 1'de olduğu gibi seçelim. 
European Journal of Science and Technology

Tablo 1. Verilerin eşit aralıkl olacak şekilde seçilmesi

\begin{tabular}{c|c|c|c|c|c|c|c|c|c|c}
\hline$j$ & 0 & 1 & 2 & 3 & 4 & 5 & 6 & 7 & 8 & 9 \\
\hline$x_{j}$ & $x_{0}=1$ & $x_{1}=5$ & $x_{2}=9$ & $x_{3}=13$ & $x_{4}=17$ & $x_{5}=21$ & $x_{6}=25$ & $x_{7}=29$ & $x_{8}=33$ & $x_{9}=37$ \\
\hline$f_{j}$ & 4208 & 5560 & 6202 & 4047 & 4585 & 3037 & 4697 & 2666 & 3047 & 2644 \\
\hline Gün & 18 Mart & 22 Mart & 26 Mart & 30 Mart & 3 Nisan & 7 Nisan & 11 Nisan & 15 Nisan & 19 Nisan & 23 Nisan \\
\hline
\end{tabular}

Bu durumda,

$p_{9}\left(x_{j}\right)=f_{j}, j=0, \ldots, 9$

şeklinde interpolasyon şartlarını sağlayan tek bir

$p_{9}(x)=\sum_{i=0}^{9} a_{i} x^{i}$ interpolasyon polinomu vardır. Buradaki $a_{i}$ katsayılarını ise, Denklem (3)'teki gibi oluşacak Vandermonde sistemini çözerek elde ederiz. Bu durumda elde edilen yaklaşım polinomu (kırmızı eğri) ve seçilen günler (sarı sütunlar) açık bir şekilde Şekil 1'de gösterilmiştir. Runge olgusu sebebiyle, grafiğin başlangıç ve bitiş noktaları arasında oldukça büyük hata sıçramaları olduğu görülmektedir. Bu hataların büyüklüklerini daha net bir şekilde Şekil 2'deki grafikte görmekteyiz. Buna göre, vaka tahmin sayılarının pozitif olduğu günler dikkate alındığında, en büyük hata 36-ıncı günde oluşur. Dahası, gerçekte 3370 olan vaka sayısı bu yaklaşım ile yaklaşık 11007 farkla 14377 olarak olarak hesaplanır.

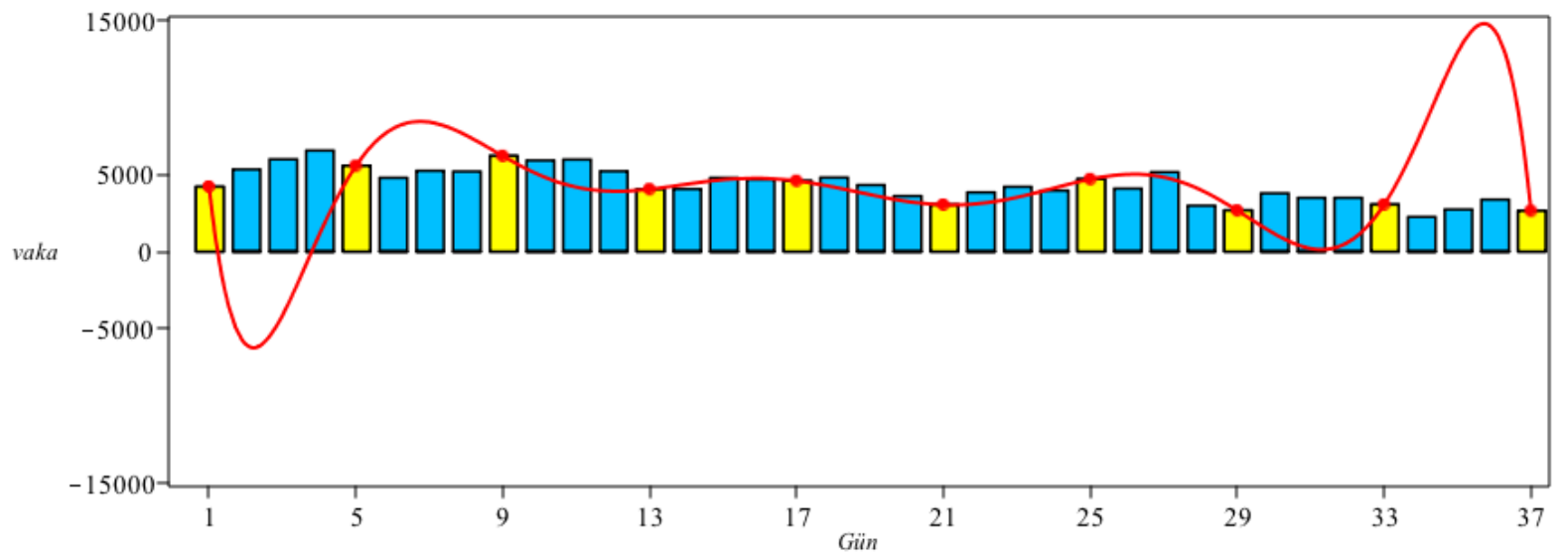

Şekil 1.37 günlük vakadan 10 tanesinin eşit aralıklı olarak seçilmesiyle elde edilen interpolasyon polinomu

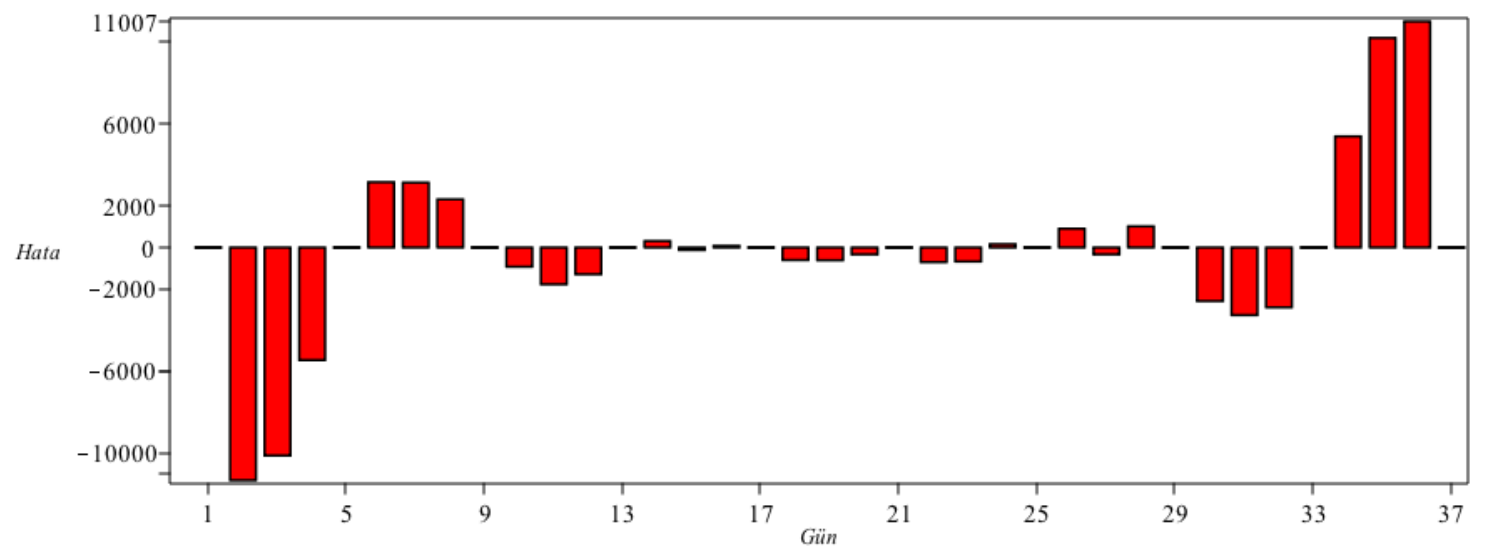

Şekil 2. Interpolasyon polinomu ile elde edilen değerler ile gerçek değerler arasındaki farklar 
Elde edilen yaklaşımın hatasını azaltmak için, şimdi ise, 10 günlük veriyi 37 günlük veri içerisinden Algoritma 1 ile Tablo 2' de olduğu gibi seçelim.

Tablo 2. Verilerin Algoritma 1 ile seçilmesi

\begin{tabular}{c|c|c|c|c|c|c|c|c|c|c}
\hline$j$ & 0 & 1 & 2 & 3 & 4 & 5 & 6 & 7 & 8 & 9 \\
\hline$x_{i}$ & $x_{0}=1$ & $x_{1}=2$ & $x_{2}=5$ & $x_{3}=10$ & $x_{4}=16$ & $x_{5}=22$ & $x_{6}=28$ & $x_{7}=33$ & $x_{8}=36$ & $x_{9}=37$ \\
\hline$f_{i}$ & 4208 & 5324 & 5560 & 5907 & 4669 & 3834 & 2973 & 3047 & 3370 & 2644 \\
\hline Gün & 18 Mart & 19 Mart & 22 Mart & 27 Mart & 2 Nisan & 8 Nisan & 14 Nisan & 19 Nisan & 22 Nisan & 23 Nisan \\
\hline
\end{tabular}

Bu durumda, yine toplamda 10 farklı nokta kullandığımızdan dokuzuncu dereceden bir polinom elde ederiz. Seçilen günler ve elde edilen interpolasyon polinomu Şekil 3'teki, oluşan interpolasyon hataları ise Şekil 4'teki gibidir. Bu grafikler incelendiğinde, bir öncekine göre oluşan hatanın büyük ölçüde azaldığı ve tüm vaka tahminlerinin de pozitif sayı olduğu görülür. Dahası, en büyük hatanın 1981 farkla 27-inci günde oluştuğu yani gerçekte 5153 vaka varken bu yaklaşım ile 3172 olarak tahmin edileceği görülür.

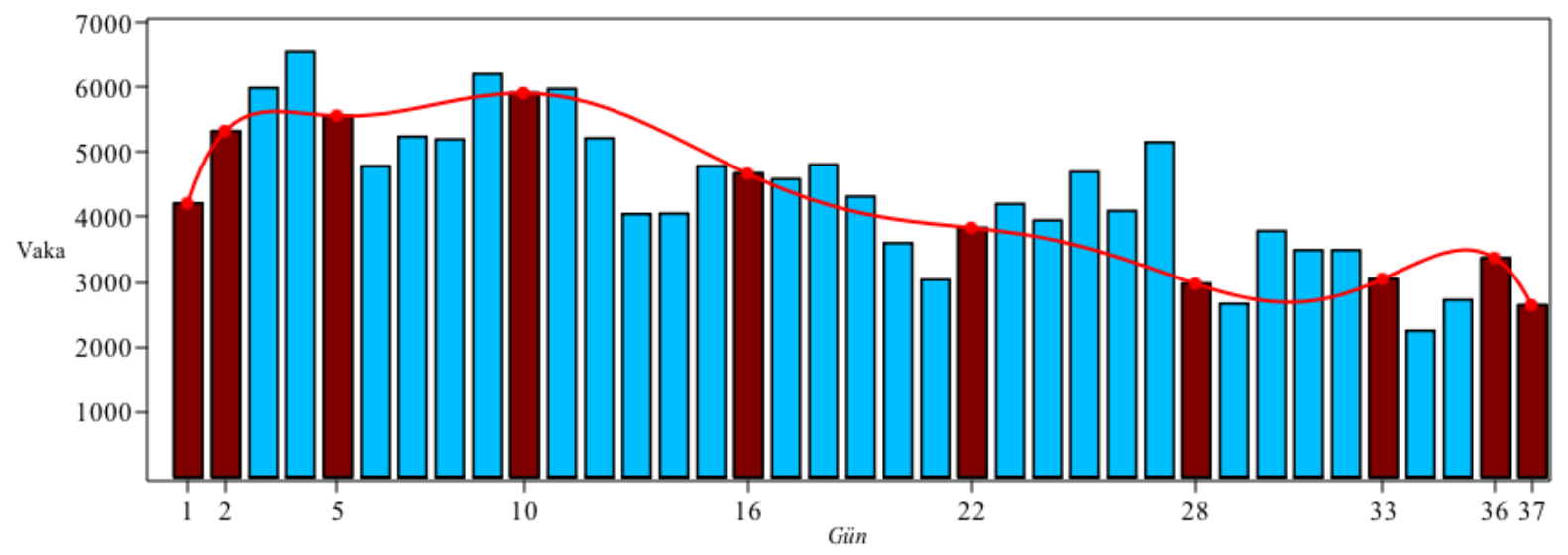

Şekil 3. 37 günlük vakadan 10 tanesinin Algoritma 1'e göre seçilmesiyle elde edilen interpolasyon polinomu

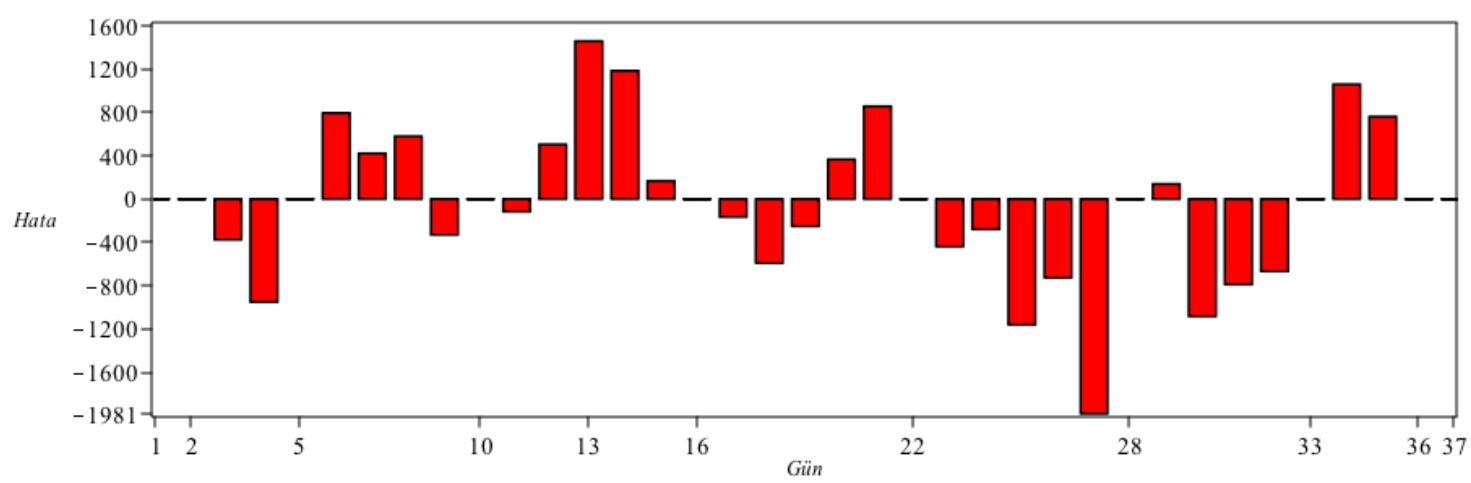

Şekil 4. Interpolasyon polinomu ile elde edilen değerler ile gerçek değerler arasındaki farklar

Başka bir açıdan, bu 37 günlük zaman diliminde her gün değil de sadece 10 gün seçilip bu günlerde test yapılmış ve kalan diğer 27 günlük vakalar ise tahmin edilmek istenseydi, eşit aralıklı gün seçimine göre yapılacak maksimum bağıl hatanın büyüklüğ̈̈ Algoritma 1 ' in kullanılması ile elde edilenin yaklaşık 8,5 katı olacaktı. Ayrıca, her iki seçimde de monomial bazlardan dolayı oluşan 
Vandermonde matrislerinin durum sayıları yüksek mertebeden olsa da, eşit aralıklı seçimde oluşan durum sayısı Algoritma 1 ile elde edilenin yaklaşık üç katıdır.

\section{Sonuç}

$\mathrm{Bu}$ çalışmada, polinom interpolasyonda eşit aralıklı noktaların kullanılması durumunda her zaman düzgün bir yaklaşımın elde edilemeyeceği gösterildi. $\mathrm{Bu}$ eşit aralıklı noktaların kötü sonuçlarından kaçınmak için önerilen mockChebysev noktalarını $[a, b]$ reel ekseninde hesaplayan hızlı bir algoritma verildi. $\mathrm{Bu}$ algoritma ile elde edilen noktalara karş11ık gelen günlerdeki veriler kullanılarak, Kovid-19 vakalarını ara günlerde tahmin etmek için Vandermonde matrisi kullanılarak interpolasyon polinomu elde edildi. Bulunan sonuçlar, günlerin eşit aralıklı olacak şekilde seçilmesi durumunda elde edilecek sonuçlar ile karşılaştırıldı. Bu karşılaştırmada, eşit aralıklı günler için çok büyük interpolasyon hatası elde edilirken, önerilen algoritma ile elde edilen günler kullanıldığında ise polinom interpolasyonda optimale yakın sonuç veren Chebysev noktalarına benzer şekilde, bu hatanın oldukça az olduğu görüldü. Ayrıca, verilen algoritma ile elde edilen noktaların kullanılması durumunda Vandermonde matrisinin durum sayısının azaldığı görüldü.

\section{Kaynakça}

[1] Boyd, J. P., \& Xu, F. (2009). Divergence (Runge phenomenon) for least-squares polynomial approximation on an equispaced grid and Mock-Chebyshev subset interpolation. Applied Mathematics and Computation, 210(1), 158-168.

[2] Boyd, J. P., \& Ong, J. R. (2009). Exponentially-convergent strategies for defeating the Runge phenomenon for the approximation of non-periodic functions, Part I: Single-interval schemes, Commun. Comput. Phys, 5(2-4), 484-497.

[3] Davis, P.J., (1963). Interpolation and Approximation, Blaisdell Publishing Co. Ginn and Co., New York.

[4] Gautschi, W. (1962). On inverses of Vandermonde and confluent Vandermonde matrices. Numerische

Mathematik, 4(1), 117-123.

[5] Gautschi, W. (1974). Norm estimates for inverses of Vandermonde matrices. Numerische Mathematik, 23(4), 337 347.

[6] Gautschi, W. (1983). The condition of Vandermonde-like matrices involving orthogonal polynomials. Linear algebra and its applications, 52, 293-300.

[7] Ibrahimoglu, B.A., (2016). Lebesgue functions and lebesgue constants in polynomial interpolation, J. Inequal. Appl. 2016 (1) 93.

[8] Ibrahimoglu, B.A., (2020). A fast algorithm for computing the mock-Chebyshev nodes, J. Comput. Appl. Math. Vol.373, 112336.

[9] Meyer, C. D. (2000). Matrix analysis and applied linear algebra(Vol. 71). Siam.

[10] Runge, C. (1901). Uber empirische Funktionen und die Interpolation zwischen aquidistanten Ordinaten, Z. Math. Phys., 46, 224-243.

[11] Trefethen, L.N., (2000). Spectral Methods in MATLAB, SIAM, Philadelphia.

[12] https:/www.worldometers.info/coronavirus/country/italy/ 